\title{
Focus on gastrointestinal tumors
}

\author{
Wolfgang Eisterer
}

Received: 6 September 2013 / Accepted: 6 September 2013 / Published online: 25 September 2013

(C) Springer-Verlag Wien 2013

Gastrointestinal cancer represents the most common tumor entity in women and men in Western Europe. There exists an enormous gap of knowledge concerning molecular biology, as well as a gap of treatment options between different gastrointestinal tumor types. While in colorectal cancer a number of substantial therapeutic improvements have been made possible by new discoveries in molecular biology within the past 10 years, there is only limited progress in the therapy of esophageal cancer, on the other hand.

Gerald Prager [1] sets his focus on the actual impact of molecular therapy in the treatment of colorectal cancer and speculates which targets might qualify for further molecular driven treatment strategies. Thomas Ruhstaller and Michael Stahl [2] summarize current treatment modalities and update the latest innovations in the treatment of locally advanced esophageal cancer.

Moreover, therapeutic "heavy weights", such as surgery, radiation, and systemic therapy innovations have been introduced to the therapeutic armamentarium by pure improvement of technology. One of those is radiofrequency ablation. Gerlig Widmann and Reto Bale [3] present advantages and limitations of this technique which, in the form of stereotactic-guided radiofrequency ablation, is used uniquely in Innsbruck.

All three manuscripts cover a broad spectrum of actual developments and discussions in the treatment of gastrointestinal tumors. I wish all readers an informative and exciting update on this topic.

\section{Conflict of interest}

The author declares that there is no conflict of interest.

\section{References}

1. Prager G. Molecular therapies of colorectal cancer: where will we go from here? Memo 2013;6. doi:10.1007/ s12254-013-0108-6.

2. Ruhstaller Th, Stahl M. Controversial issues in the therapy of oesophageal carcinoma. Memo 2013;6. doi:10.1007/ s12254-013-0096-6.

3. Widmann G, Bale R. Radiofrequency ablation in gastrointestinal cancer: obstacles and goals. Memo 2013;6. doi: 10.1007/s12254-013-0097-5.

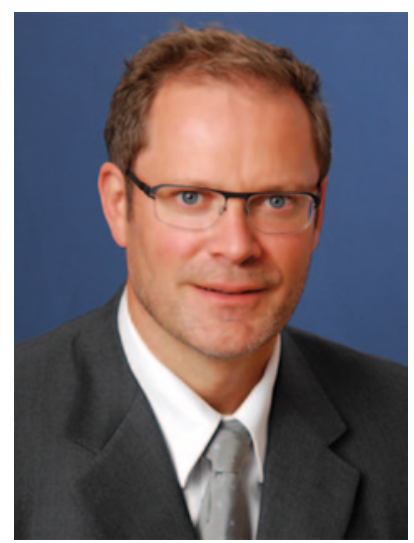

Univ.-Prof. Dr. W. Eisterer $(\square)$

Medical University Innsbruck, Internal Medicine V,

Anichstrasse 35,6020 Innsbruck, Austria

e-mail: wolfgang.eisterer@i-med.ac.at 